\title{
Evaluation of PPP2R5C gene expression in Iranian patients with B-Acute lymphoblastic leukemia and its association with clinical and laboratory findings
}

Esmaeil Rostami ${ }^{1}$, Hossein Ayatollahi ${ }^{2}$, Hassan boustani ${ }^{3}$, Abbas Ghotaslou ${ }^{3 *}$, Mohammad Hadi Sadeghian ${ }^{4}$, Mohammad Reza Keramati ${ }^{4}$, Elahe Zeinali ${ }^{5}$, Abolfazl Rad ${ }^{6}$

1. Department of Laboratory Sciences, Faculty of Paramedicine, Sabzevar University of Medical Sciences, Sabzevar, Iran

2. Cancer Molecular Pathology Research Center, Department of Hematology and Blood Bank, Faculty of Medicine, Mashhad University of Medical Sciences, Mashhad, Iran

3. Department of Hematology, School of Allied Medical Sciences, Iran University of Medical Sciences, Tehran, Iran

4. Neonatal Research Center, Imam Reza Hospital, Faculty of Medicine, Mashhad University of Medical Sciences, Mashhad, Iran

5. Department of laboratory sciences, Faculty of Paramedicine, Mashhad University of Medical Sciences, Mashhad, Iran

6. Department of Biochemistry and Nutrition, Cellular and Molecular Research Center, Sabzevar University of Medical Sciences, Sabzevar, Iran

*Corresponding author:Tel: +98 9224817656 Fax: +98

Address: Abbas Ghotaslou, Department of Hematology, School of Allied Medical Sciences, Iran University of Medical Sciences, Tehran, Iran

E-mail: a.ghotaslou@gmail.com

Received; 2017/02/22 revised; 2017/04/4 accepted; 2017/04/21

\section{Abstract}

Introduction: PPP2R5C is one of the regulatory $\mathrm{B}$ subunits of protein phosphatase $2 \mathrm{~A}$ (PP2A), which is a tumor suppressor. PPP2R5C plays a critical role in cell proliferation, differentiation, and transformation. Considering these vital functions, we investigate the gene expression in Iranian patients with B-Acute Lymphoblastic Leukemia (B-ALL) and its association with clinical and laboratory finding.

Materials and methods: In this case-control study, peripheral blood samples were collected from 60 B-ALL patients and 30 healthy controls. PPP2R5C expression levels were determined by Real-time PCR. After calculation of CT for target and control genes, we calculated $\Delta \mathrm{CT}$. Finally we compared the PPP2R5C expression levels in patients with control group.

Results: Significantly higher expression of PPP2R5C was found in the B-ALL patients $(2.15 \pm 2$. 50) compared with control group. There was no correlation between PPP2R5C expression and clinical and laboratory findings and FAB (French-American-British) subtype of patients.

Conclusion: we demonstrated PPP2R5C overexpression in B-ALL patients. Although there was no significant correlation between PPP2R5C expression, clinical and laboratory finding and also with FAB subtypes of patients.

Keywords: B-Acute Lymphoblastic Leukemia, PPP2R5C, PP2A, Gene expression

\section{Introduction}

Acute Lymphoblastic Leukemia (ALL) is a form of leukemia that is characterized by overproduction and subsequently bone marrow infiltration of lymphoblasts and other body organs involvement. Although it may be diagnosed at any age, it is the most common malignancy of infants and teenagers. In the current WHO 
classification, two subtypes, B-ALL and T-ALL, are recognized; B-ALL comprises the great majority of cases (1). In many cases the cause of leukemia is ambiguous but mutations, deletions, chromosomal defects, up or down regulation of cellular signaling pathways as well as dysregulation of Epigenetic events can be causative factors of leukemia (2-4). The opposing function of phosphatases and kinases is a more important mechanism involved in regulation of different signaling pathways, so aberrations in regulatory proteins of these mechanisms may be from significant causes of leukemia (5).

Protein phosphatase 2A (PP2A) is a Ser/Thr protein phosphatase with a heterotrimer structure. It has a catalytic subunit (C), a scaffold subunit (A) and several variable regulatory subunits (B). The regulatory subunits are four families which B56 family members consist of PPP2R5A, PPP2R5B, PPP2R5C, PPP2R5D, and PPP2R5E. Observations demonstrated that B56 family are more important in malignant transformation (6$10)$, by its inducing effect on dephosphorylation of P53. PPP2R5C plays a vital role in cell proliferation, differentiation, and transformation (11-13). With regard to the critical functions of PPP2R5C we investigated the gene expression of this protein in Iranian patients with B-Acute Lymphoblastic Leukemia and its association with clinical and laboratory findings.

\section{Materials and methods}

This study was performed in the Research laboratory of molecular hematopathology of Ghaem Hospital in Mashhad, Iran. 60 newly diagnosed and untreated patients with B-ALL and 30 individuals as control were enrolled into the study. All patients were categorized in FAB classification groups. At the beginning of study, informed consent was obtained from all groups. The clinical and laboratory parameter including lymphadenopathy, splenomegaly,hepatomegaly, demographic data, immunophenotyping results and other laboratory parameters, were retrieved from medical records of patients.

Peripheral blood was collected from all patients in K2-EDTA containing vials. Ficoll gradient centrifugation was used to isolate mononuclear cells. The RNA was extracted using RNX plus kit according to the manufacturer's instructions (Cinnagen, Tehran, Iran). Subsequently, cDNA synthesis was performed using primers of Random Hexamer. PPP2R5C gene expression analysis on patient's samples and control group was done using real time-PCR method. Gene expression levels, were applied by SYBRGreen (TAKARA) and relative expression was calculated from $2^{-\Delta \Delta C T}$ method. PPP2R5C gene expression normalized against the internal gene, beta-2-microglobolin $(\beta 2 \mathrm{M})$, by specific primers depicted in table 1 .

In order to ensure the specificity of the real-time PCR products, melting curves were plotted after each working run. For standard curve, 0.1, 0.01, and 0.001 dilutions were prepared from primers of PPP2R 5C and $\beta 2 \mathrm{M}$. Amplification curves were plotted following performing of the test. The results of PPP2R5C gene expression levels in patients were compared with that of the control group. Data were analyzed by SPSS, version19. The Kolmogorov-Smirnov test and ChiSquare independent sample t-test were used in case needed. P-value less than 0.05 were considered as significant.

\section{Results}

We studied 60 patients diagnosed with BALL and 30 individuals as control group. The patients group included 30(50\%) males, 30(50\%) females and the control group was made of $15(50 \%)$ males and $15(50 \%)$ females. Mean \pm SD age of patients and control group were $13 \pm 14$ years (range: $1-65$ years) and $16 \pm 7$ years (range: 3-42 years), respectively. FAB classification of patients include $45(75 \%)$, ALL-L1 and 15(25\%) ALL-L2 
respectively. WBC counts, platelet counts and hemoglobin concentration in patients and the correlation between FAB classification and gene expression level in patients are shown in table 2 . There was no significant relationship between the expressions level of PPP2R5C and these parameters. The included clinical features were hepatomegaly (44\%), splenomegaly (66\%), lymphadenopathy (42\%) and petechia-purpura $(24 \%)$. There was no significant association between the expressions level 3of PPP2R5C and clinical presentations (Table 3 ).
We examined the genetic abnormalities such as $\mathrm{t}(12 ; 21), \mathrm{t}(1 ; 19)$ and $\mathrm{t}(9 ; 22)$, there was no significant correlation between the expressions level of PPP2R5C and genetic abnormalities (Table 4). Significantly higher expression of PPP2R5C was found in the patients $(2.15$ \pm 2.50 ) compared with healthy controls. The number of patients that had upregulated expression of PPP2R5C was $70 \%$ (42 out of 60). There was no significant relationships between the expressions level of PPP2R5C and different FAB subtypes ( $\mathrm{P}$ value $>0.05$ ).

Table 1. Primer sequence for two genes (PPP2R5C and $\beta 2 \mathrm{M})$.

\begin{tabular}{lll}
\hline Gene & Primer & Sequence (5' to 3') \\
\hline PPP2R5C & Forward & GTAATAAAGCGGGCAG-CAGG \\
& Reverse & CAAAGTCAAAGAGACGCAACA \\
\hline$\beta 2 \mathrm{M}$ & Forward & CAGCAAGGACTGGTCTTTCTAT \\
& Reverse & CAGCAAGGACTGGTCTTTCTAT \\
\hline
\end{tabular}

Table 2. Characteristics and laboratory findings of the patients with B-ALL.

\begin{tabular}{|c|c|c|c|c|c|}
\hline \multirow[t]{2}{*}{ Characteristics } & \multicolumn{2}{|c|}{ Fab subtype } & \multicolumn{2}{|c|}{ PPP2R5C Expression Level } & \multirow[b]{2}{*}{$\mathrm{P}$ value } \\
\hline & L1 & $\mathrm{L} 2$ & Up Regulate & Down Regulate & \\
\hline Number of Patients, (\%) & $45(75)$ & $15(25)$ & $42(70)$ & $18(30)$ & \\
\hline Male & 22 & 8 & 20 & 11 & \\
\hline Female & 23 & 7 & 22 & 7 & \\
\hline $\begin{array}{l}\text { WBC count }\left(10^{9} / \mathrm{L},\right. \\
\text { median })\end{array}$ & 51.05 & 62.16 & 57.55 & 49.14 & 0.641 \\
\hline $\begin{array}{l}\text { Platelet count }\left(10^{9} / \mathrm{L} \text {, }\right. \\
\text { median })\end{array}$ & 79.86 & 11.84 & 73.07 & 55.31 & 0.192 \\
\hline $\mathrm{Hb}, \mathrm{g} / \mathrm{dl}$ (median) & 6.5 & 7.2 & 6.42 & 7.08 & 0.383 \\
\hline
\end{tabular}

Table 3. Differences in expression level of PPP2R5C in patients with different clinical presentations.

\begin{tabular}{lcccccccc}
\hline Clinical & \multicolumn{2}{c}{ Lymphadenopathy } & \multicolumn{2}{c}{ Splenomegaly } & \multicolumn{2}{c}{ Hepatomegaly } & \multicolumn{2}{c}{ Petechiae-Purpura } \\
\cline { 2 - 8 } presentations & Positive & Negative & Positive & Negative & Positive & Negative & Positive & Negative \\
\hline PPP2R5C level & 3.06 & 1.50 & 2.10 & 2.25 & 2.52 & 1.93 & 3.47 & 1.74 \\
P value & 0.227 & \multicolumn{2}{c}{0.757} & & 0.418 & 0.118 \\
\hline
\end{tabular}

Table 4. Differences in expression level of PPP2R5C in B- ALL patients with different genetic abnormalities.

\begin{tabular}{lcccccc}
\hline Translocation & \multicolumn{2}{c}{$\mathrm{t}(12 ; 21)$} & \multicolumn{2}{c}{$\mathrm{t}(9 ; 22)$} & \multicolumn{2}{c}{$\mathrm{t}(1 ; 19)$} \\
\cline { 2 - 7 } & Positive & Negative & Positive & Negative & Positive & Negative \\
\hline Number & 11 & 49 & 17 & 43 & 7 & 53 \\
PPP2R5C level & 2.476 & 2.069 & 2.547 & 1.958 & 2.683 & 2.073 \\
P value & & 0.639 & & 0.488 & & 0.550 \\
\hline
\end{tabular}

\section{Discussion}

PPP2R5C is one of the regulatory subunits of protein phosphatase $2 \mathrm{~A}$ (PP2A) which is a key cellular serine/threonine phosphatase affecting the phosphorylation site of many proteins (9). PP2A is one of the Ras-Raf signaling pathway regulators. The normal function of Akt is tightly modulated by phosphorylation events (14). The function of PP $2 \mathrm{~A}$ in $\mathrm{Wnt} / \beta$-catenin signaling is similar to its role in MAPK pathway (15). In response to $\gamma$ radiation PP2A dephosphorylates p53 hence affecting apoptosis in mammalian cells [16]. PP2A also plays a prominent role in 
controlling the accumulation of the protoncoprotein, c-Myc (17).

Recent studies have demonstrated decreased expression of PPP2R5C in BCLL, so it was concluded to be a marker of progressive disease in B-CLL. Researchers have compared expression of PPP2R5C in stable and progressive B-CLL without a control group (18). Another study, demonstrated that overexpression of PPP2R5C gene in ALL patients compared with control group (19). Two different mutations in lung cancer and intestinal cancer can disrupt B56 $\gamma$-P53 interaction $(12,20)$.

It has been showed that PPP2R5C suppression by RNAi can inhibit the proliferation of the Molt4 and Jourkat $\mathrm{T}$ cells (21). It also has been showed that the proliferation of the PPP2R5C-siRNA treated CML cell line was decreased in K562 Cell line (22). In our study significantly higher expression of PPP2R5C was found in Iranian patients with the B-ALL. We studied more patients than previous studies to confirm overexpression of PPP2R5C and also examined the relation of the PPP2R5C expression with clinical and laboratory findings that there was no significant relationship. Additionally, we also examined expression level of PPP2R5C

\section{References}

1. Jabbour E, O’Brien S, Konopleva M, Kantarjian H. New insights into the pathophysiology and therapy of adult acute lymphoblastic leukemia. Cancer.2015; 121(15): 2517-28.

2. Bacher U, Kohlmann A, Haferlach T. Gene expression profiling for diagnosis and therapy in acute leukaemia and other haematologic malignancies. Cancer Treat Rev. 2010; 36(8):637-46.

3. Oshiro A, Tagawa H, Ohshima K, Karube K, Uike N, Tashiro Y, et al. Identification of subtype-specific genomic alterations in aggressive adult among FAB subtypes of B-ALL (L1, L2) that there was no significant difference in expression level between them. In regard with the importance of PP2A in cancer, as demonstrated in several studies, expression pattern of PPP2R5C might be a critical finding in ALL patients. Definitive results for proven the upregulation of PPP2R5C in B-ALL patients particularly relation of the expression level of PPP2R5C with clinical and laboratory findings could be demonstrated by larger number of samples studied.

\section{Conclusion}

The present study revealed PPP2R5C overexpression in B-ALL patients. Although there was no significant relation between PPP2R5C expression clinical and laboratory finding and also with FAB subtypes of patients.

\section{Acknowledgments}

The authors gratefully acknowledge the scientific and technical support of colleagues at the Ghaem hospital. This study supported by a grant from the Advice Chancellor of the Mashhad University of Medical Sciences.

T-cell leukemia/lymphoma. Blood. 2006; 107(11):4500-7.

4. Hamalainen MM, Kairisto V, Juvonen V, Johansson J, Auren J, Kohonen K, et al. Wilms tumour gene 1 overexpression in bone marrow as a marker for minimal residual disease in acute myeloid leukaemia. Eur J Haematol. 2008; 80(3):201-7.

5. Haendeler J, Hoffmann J, Rahman S, Zeiher AM, Dimmeler S. Regulation of telomerase activity and anti-apoptotic function by protein-protein interaction and phosphorylation. FEBS Lett. 2003; 536(1-3):180-6. 
6. Yang J, Phiel C. Functions of B56containing PP2As in major developmental and cancer signaling pathways. Life Sci. 2010; 87(2326):659-66.

7. Arroyo JD, Hahn WC. Involvement of PP2A in viral and cellular transformation. Oncogene. 2005; 24(52):7746-55.

8. Chen W, Possemato R, Campbell KT, Plattner CA, Pallas DC, Hahn WC. Identification of specific PP2A complexes involved in human cell transformation. Cancer Cell. 2004; 5(2):127-36.

9. Muneer S, Ramalingam V, Wyatt $\mathrm{R}$, Schultz RA, Minna JD, Kamibayashi C. Genomic organization and mapping of the gene encoding the PP2A B56 $\gamma$ regulatory subunit. Genomics. 2002; 79(3):344-8.

10. Lee TY, Lai TY, Lin SC, Wu CW, Ni IF, Yang YS, et al. The B56gamma3 regulatory subunit of protein phosphatase 2A (PP2A) regulates $\mathrm{S}$ phase-specific nuclear accumulation of PP2A and the G1 to S transition. J Biol Chem. 2010; 285(28):21567-80.

11. Shouse GP, Cai X, Liu X. Serine 15 phosphorylation of p53 directs its interaction with B56gamma and the tumor suppressor activity of B56gamma-specific protein phosphatase 2A. Mol Cell Biol. 2008; 28(1):448-56.

12. Shouse GP, Nobumori Y, Liu X. A B56gamma mutation in lung cancer disrupts the p53-dependent tumorsuppressor function of protein phosphatase 2A. Oncogene. 2010; 29(27):3933-41.

13. Perrotti D, Neviani P. ReSETting PP2A tumour suppressor activity in blast crisis and imatinib-resistant chronic myelogenous leukaemia. $\mathrm{Br} \mathrm{J}$ Cancer. 2006; 95(7):775-81.

14. Sarbassov DD, Guertin DA, Ali SM, Sabatini DM. Phosphorylation and regulation of $\mathrm{Akt} / \mathrm{PKB}$ by the rictor-
mTOR complex. Science. 2005; 307(5712):1098-101.

15. Zhang W, Yang J, Liu Y, Chen X, Yu $\mathrm{T}$, Jia J, et al. PR55 alpha, a regulatory subunit of PP2A, specifically regulates PP2A-mediated beta-catenin dephosphorylation. J Biol Chem. 2009; 284(34):22649-56.

16. Li HH, Cai X, Shouse GP, Piluso LG, Liu X. A specific PP2A regulatory subunit, B56gamma, mediates DNA damage-induced dephosphorylation of p53 at Thr55. Embo J. 2007; 26(2):402-11.

17. Yeh E, Cunningham $\mathrm{M}$, Arnold $\mathrm{H}$, Chasse D, Monteith T, Ivaldi G, et al. A signalling pathway controlling cMyc degradation that impacts oncogenic transformation of human cells. Nat Cell Biol. 2004; 6(4):308-18.

18. Falt S, Merup M, Gahrton G, Lambert B, Wennborg A. Identification of progression markers in B-CLL by gene expression profiling. Exp Hematol. 2005; 33(8):883-93.

19. Zheng H, Chen Y, Chen S, Niu Y, Yang L, Li B, et al. Expression and distribution of PPP2R5C gene in leukemia. J Hematol Oncol. 2011; 4(21):1756-8722.

20. Loveday C, Tatton-Brown K, Clarke M, Westwood I, Renwick A, Ramsay $\mathrm{E}$, et al. Mutations in the PP2A regulatory subunit $\mathrm{B}$ family genes PPP2R5B, PPP2R5C and PPP2R5D cause human overgrowth. Hum Mol Genet. 2015; 24(17):4775-9.

21. Chen Y, Liu S, Shen Q, Zha X, Zheng $\mathrm{H}$, Yang L, et al. Differential gene expression profiles of PPP2R5CsiRNA-treated malignant T cells. DNA Cell Biol. 2013; 32(10):573-81.

22. Shen Q, Liu S, Chen Y, Yang L, Chen $\mathrm{S}, \mathrm{Wu} \mathrm{X}$, et al. Proliferation inhibition and apoptosis induction of imatinibresistant chronic myeloid leukemia cells via PPP2R5C down-regulation. J Hematol Oncol. 2013; 6(64):17568722. 